\title{
Neurosyphilis revealed by compressive cervical spine syphilitic gumma: a case report
}

\author{
Anasse Mejdoubi ${ }^{1} \cdot$ Mohamed Khoulali $^{1} \cdot$ Nabil Raouzi $^{1} \cdot$ Siham Nasri ${ }^{2} \cdot$ Yassine Mebrouk $^{3} \cdot$ Noureddine Oulali $^{1}$. \\ Fayçal Moufid ${ }^{1}$
}

Received: 13 April 2020 / Revised: 13 June 2020 / Accepted: 16 June 2020

(C) International Spinal Cord Society 2020

\begin{abstract}
Introduction Neurosyphilis is a sexually transmitted disease secondary to the invasion of the central nervous system by the Treponema pallidum. The spinal syphilitic gumma is rare.

Case presentation We report a case of extradural cervical spinal syphilitic gumma revealed by spinal cord compression in a 58-year-old male. The epidural lesion was removed via a posterior approach. Histological examination revealed syphilis. Syphilis serologies were positive. Brain MRI showed an associated cerebro-meningeal syphilitic gumma. Antibiotic regime based on aqueous penicillin $\mathrm{G}$ was introduced for 14 days.

Discussion Currently, there is an increase in the frequency of syphilis and changes in its clinical manifestations. Neurosyphilis can take atypical forms. Spinal syphilitic gumma is a rare manifestation and its association with cerebral involvement is exceptional. Diagnosis is based on serologies in the blood and cerebrospinal fluid. The place of imagery, especially magnetic resonance imaging, is essential. Neurosyphilis should be discussed as a possible differential diagnosis in evaluation of spinal and cerebral lesions.
\end{abstract}

\section{Introduction}

Neurosyphilis includes all neurological disorders secondary to the invasion of the nervous system by the Treponema pallidum. It can appear during the primo-secondary (early neurosyphilis) or tertiary (late neurosyphilis) phases. The diagnosis of neurosyphilis is currently challenging because of the diversity of symptoms and atypical presentations. Furthermore, the widespread use of penicillin has altered the clinical manifestations of neurosyphilis and serological profiles, making diagnosis even more difficult $[1,2]$.

Anasse Mejdoubi

anasse.mejdoubi@gmail.com

1 Department of Neurosurgery, University Hospital Center Mohammed VI, Faculty of Medicine, Mohammed First University, Oujda, Morocco

2 Department of Radiology, University Hospital Center Mohammed VI, Faculty of Medicine, Mohammed First University, Oujda, Morocco

3 Department of Neurology, University Hospital Center Mohammed VI, Faculty of Medicine, Mohammed First University, Oujda, Morocco
Compared to cerebral involvement, spinal syphilis is rare, manifested by myelitis, tabes dorsalis, and gummas. Spinal syphilitic gumma represents an exceptional entity; only a dozen cases have been reported in the literature [3-5]. The association of spinal and cerebral syphilitic gummas is even rarer [6]. In this article, we report an exceptional case of neurosyphilis combining an extradural cervical spinal and cerebro-meningeal syphilitic gumma revealed by spinal cord compression.

\section{Case report}

A 58-year-old married male, with a 2-month history of progressive heaviness in the four limbs was admitted to a peripheral hospital. Due to worsening of the neurological deficit and the appearance of genito-sphincter dysfunction, the patient was referred to our hospital center for management. Physical examination in the emergency room revealed a conscious male, well oriented, without disturbance of the superior functions, or cranial nerve palsy. Neurological examination revealed spastic tetraplegia with muscle strength $2 / 5$ in the upper limbs and $0 / 5$ in the lower limbs with hypoesthesia below the T4 level. Spinal magnetic resonance 
Fig. 1 Cervical spinal MRI.

a Sagittal T1-weighted image shows a posterior hypointense lesion next to C5. b Sagittal T2weighted image shows a hypointense lesion. c Sagittal T1-weighted gadoliniumenhanced image shows homogeneous enhancement. d Axial T2-weighted image and e axial T1-weighted gadoliniumenhanced image shows a lesion occupying the posterior epidural space compressing the spinal cord.
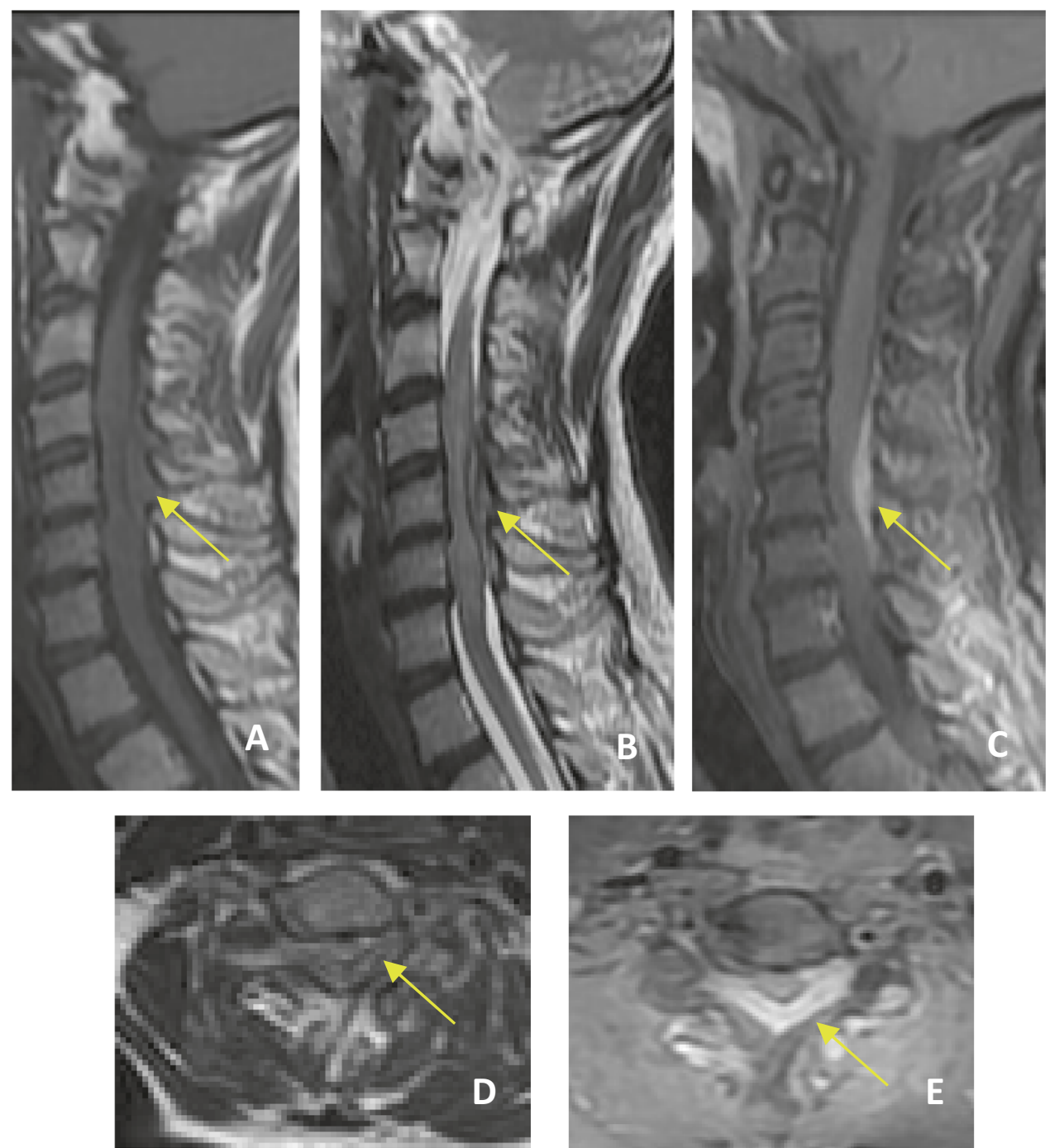

imaging (MRI) showed (Fig. 1) posterior cervical epiduritis on the 5th cervical vertebra (C5), hypointense on T1-weighted image, hypointense on T2-weighted image, enhancing after injection of gadolinium, exerting a mass effect on the cervical cord. The preoperative biological assessment showed a lymphocytic hyperleukocytosis $84 \%$ (leukocyte $=16,280$, with normal level: $4000-10,000 / \mathrm{mm}^{3}$ ) and C-reactive protein at $0.9 \mathrm{mg} / \mathrm{l}$ (normal level: $0.00-5.00 \mathrm{mg} / \mathrm{l}$ ). An emergency posterior laminectomy of C5 was performed without osteosynthesis. A thick extradural lesion adhering to the dura was separated and extirpated (Fig. 2). Histopathological examination revealed a chronic inflammatory epiduritis rich in plasmocyte elements with Russel's body evoking a syphilitic gumma. Given this result, we performed syphilitic serology in the blood which showed a reactive Veneral Disease Reagent Laboratory (VDRL) test with a titer of $1 / 64$ and highly positive (3+) Treponema pallidum Hemagglutination Assay (TPHA). The syphilitic serology in his wife also returned positive. Screening for immunodeficiency virus antibodies

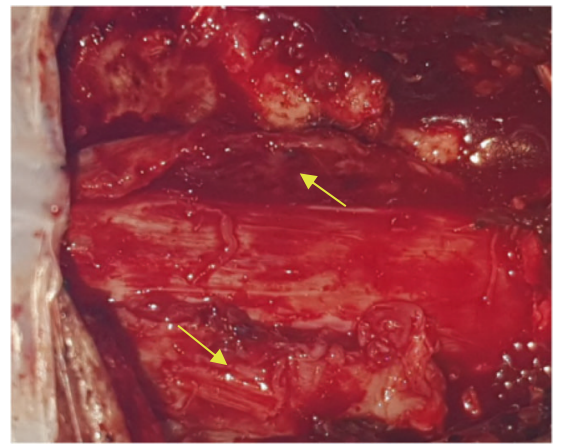

Fig. 2 Intraoperative image. Intraoperative aspect after dissection of the lesion from the dura mater.

was negative. In further questioning, the patient reported an untreated genital lesion in 2004. The current dermatological examination revealed two genital chancres. Lumbar puncture revealed clear cerebrospinal fluid (CSF), hyperproteinorachia at $3.25 \mathrm{~g} / \mathrm{l}$ (normal level: $0.15-0.45 \mathrm{~g} / \mathrm{l}$ ) with an increase in 
Fig. 3 Brain MRI.

a Hypointense nodular lesion on sagittal T1-weighted image.

b Hypointense lesion on sagittal

T2-weighted image.

c Perilesional edema on the

FLAIR sequence. d Sagittal T1-

weighted gadolinium-enhanced

image, e, $\mathbf{f}$ axial T1-weighted

gadolinium-enhanced image sh.
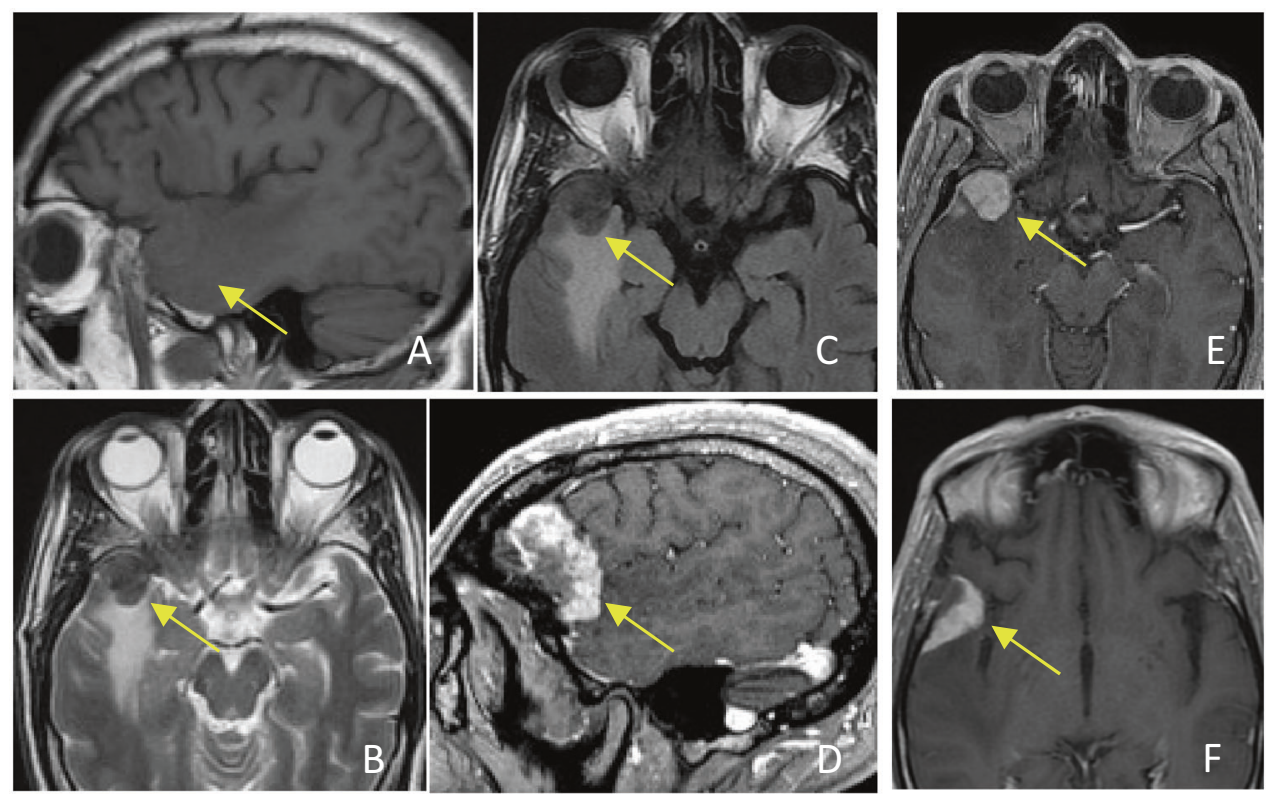

immunoglobulin $\mathrm{G}(\mathrm{IgG}=85 \mathrm{mg} / \mathrm{dl}$, with normal level: $2.00-4.00 \mathrm{mg} / \mathrm{dl}$ ) and a normal white blood cell count (1 element, with normal level $<5 / \mathrm{mm}^{3}$ ). VDRL and TPHA test in the CSF were negative while the Fluorescent Treponemal Antibody Absorption (FTA-abs) test in the CSF returned positive. A brain MRI (Fig. 3) in search of a secondary lesion objectified a right temporal cerebro-meningeal lesion, hypointense on T1-weighted image, heterogeneous hypointense on T2-weighted image, homogeneous enhancement after contrast injection and associated with a wide range of perilesional edema on FLAIR sequence evoking a cerebromeningeal syphilitic gumma with intraparenchymal extension. The patient was given IV 24 million units of aqueous penicillin $\mathrm{G}$ per day for 14 days.

The follow-up at 5 months shows a partial recovery of the sensory-motor deficit, mainly in the two upper limbs, with persistence of vesico-sphincter disorders which requires the use of a permanent urinary catheter. Due to the COVID-19 pandemic and the national containment that has restricted the circulation of the population, our patient is undergoing a reduced re-education and rehabilitation program. This will impact neurological recovery and require a longer follow-up period.

\section{Discussion}

Syphilis is a systemic sexually transmitted infection that is worldwide spread caused by a spirochete, T. pallidum. It is a very old disease that had become rare in industrialized countries since the discovery of penicillin until the 1990-2000s during which it resurfaced due to the outbreak of the HIV/AIDS infection [1, 7, 8]. Neurosyphilis is the invasion of the nervous system by $T$. pallidum; it complicates $5-10 \%$ of untreated syphilis. The prevalence of neurosyphilis is particularly high in people with HIV coinfection [7-9]. T. pallidum can infect different parts of the central nervous system such as the meninges, brain, brainstem, cerebellum, spinal cord, and nerve roots, as well as the cerebral and spinal vessels. The clinical forms of neurosyphilis are therefore diverse [10]. Neurosyphilis has two stages [11]:

(1) Early neurosyphilis: the initial stages of syphilis. Most often asymptomatic (asymptomatic neurosyphilis), the main neurological manifestations are meningitis or meningoencephalitis and cranial pair palsy [7, 8, 11, 12].

(2) Late neurosyphilis: it occurs one year after initial infection, and is often associated with vascular and/or parenchymal involvement (meningo-vascularitis, general paresis, myelitis, tabes dorsalis, and syphilitic gumma) [7, 8, 11, 13].

Currently, typical forms are becoming less common while there is an increase in the incidence of atypical manifestations such as psychiatric and cognitive disorders $[1,2]$. These changes make diagnosis more challenging, especially in the early phase.

Syphilitic gumma is the consequence of a cellular immune response, secondary to $T$. pallidum invasion, causing the formation of an inflammatory granulation "tumor-like" consisting of lymphocytes and plasma cells. This destructive lesion can occur in any part of the organism $[3,4]$. In the central nervous system, syphilitic gumma develops from the dura and pia mater [6]. Usually cerebro- 
meningeal, it may exceptionally be intracerebral posing a problem of differential diagnosis with a malignant cerebral tumor [14]. In the spine, some cases of syphilitic gumma have been reported in the literature. They can develop in the intradural, intramedullary or extramedullary space, or the extradural space as in our case $[4,15]$. The association of cerebral and spinal syphilitic gummas is exceptional. The first case of this association was reported by Shen [6].

Microbiological diagnosis of syphilis is based on a serological study in the blood which includes VDRL, TPHA, and FTA-abs. The biological diagnosis of neurosyphilis is based on nonspecific arguments (hypercellularity of the CSF, hyperproteinorachia, increase of the immunoglobulins $\mathrm{G}$ in the CSF) and serological tests in the CSF (VDRL, TPHA, TPPA, and FTA-abs). Normal CSF does not eliminate neurosyphilis $[7,8,11,13,16]$. In our case, biochemical abnormalities were found in the CSF (hyperproteinorachia and increased immunoglobulin G) with negative CSF syphilis serology.

Neuroimaging plays a fundamental role in the management of neurosyphilis. It provides diagnostic elements, ensures radiological monitoring, and assesses therapeutic effectiveness. MRI represents the most efficient technique in terms of sensitivity and specificity [17]. The spectrum of neuroradiological lesions includes cortico-subcortical parenchymal atrophy, predominant in the frontotemporal regions, lesions of white matter and gray nuclei, and infarctions distributed in the vascular territories of the perforating arteries [17-19]. Syphilitic gumma presents on MRI as a hypo or iso-intense nodule on T1-weighted image and hyperintense on T2-weighted image, with homogeneous enhancement after contrast injection, and may be accompanied by a linear enhancement of the dura mater [6, 19]. In our case, the MRI was hypointense on T1weighted image, and hypointense on T2-weighted image with homogeneous enhancement after contrast injection. However, it should be noted that the radiological features of neurosyphilis are as variable as the clinical manifestations; the lesions observed could be confused with other neurological pathologies $[17,18]$. Imaging follow-up after antisyphilitic treatment can show neuroradiological progression in some cases including infarction lesions, mild to severe brain atrophy, and white matter demyelination suggesting that we need to continue imaging follow-up and treatment of these patients [20].

First-line treatment for neurosyphilis in any form, including central nervous system gummas, is represented by highdose intravenous aqueous penicillin $\mathrm{G}$. The recommended protocol is $18-24$ million units, administered intravenously for 10-14 days [7]. In the case of penicillin allergy, ceftriaxone is an alternative treatment at $2 \mathrm{~g}$ per day in intramuscular or intravenous for 14 days. Azithromycin and doxycycline are other therapeutic alternatives for penicillin allergy $[7,16]$. Administration of $60 \mathrm{mg}$ of prednisone during the first $24 \mathrm{~h}$ of penicillin cure is recommended to prevent the Jarisch-Herxheimer reaction [7]. The same treatment protocol is used for the patients with HIV co-infection; a prophylactic treatment based on intramuscular benzathine penicillin at a dosage of 2.4 million units for 3 weeks or doxycycline $200 \mathrm{mg}$ twice a day for a month is recommended, with clinical and serological control for at least 2 years [7]. Almost all of reported spinal syphilitic gumma cases were suspected as spinal tumors and they were undergoing surgery. On the other hand, most cerebral syphilitic gumma can be significantly reduced or completely resorbed after the antisyphilis treatment, and surgery may be unnecessary [4]. Neurosurgical treatment of syphilitic gumma should be reserved in case of signs of compression (spinal cord compression, and intracranial hypertension) or secondary neurological worsening $[3,5,21]$. Early diagnosis of neurosyphilis and appropriate antibiotic treatment can show clinical improvement and resolution of CSF abnormalities [5, 8]. However, neurologic deficits may fail to improve even with proper treatment [7]. In patients with meningeal neurosyphilis, quick resolution of symptoms is the rule, whereas in parenchymatous forms, the resolution may not occur [13]. Resolution of CSF abnormalities is the best measure of the adequacy of treatment. The CDC recommends that CSF examination be repeated every 6 months after completion of therapy until abnormalities resolve [7, 8]. The follow-up of our patient at 5 months of the treatment finds a partial recovery; however, a longer-term follow-up is necessary to judge the neurological outcome.

Neurosyphilis is a major complication of syphilis and still represents a public health problem, characterized by clinical, biological, and radiological polymorphism. Atypical forms are now the most common; hence, the importance of screening and adequate treatment of all syphilis in the initial phase.

\section{Compliance with ethical standards}

Conflict of interest The authors declare that they have no conflict of interest.

Publisher's note Springer Nature remains neutral with regard to jurisdictional claims in published maps and institutional affiliations.

\section{References}

1. Mitsonis C, Kararizou E, Dimopoulos N, Triantafyllou N, Kapaki E, Mitropoulos $\mathrm{P}$, et al. Incidence and clinical presentation of neurosyphilis: a retrospective study of 81 cases. Int J Neurosci. 2008;118:1251-7.

2. Skalnaya A, Fominykh V, Ivashchenko R, Averchenkov D, Grazhdantseva L, Frigo N, et al. Neurosyphilis in the modern era: Literature review and case series. J Clin Neurosci. 2019;69:67-73. 
3. Salem KMI, Majeed H, Bommireddy R, Klezl Z. Tertiary syphilis in the cervical spine: a case report and review of the literature. Glob Spine J. 2013;3:041-6.

4. Cui L, Xu Z, Hou H. Diagnosis and treatment of spinal syphilitic gumma: a case report. Front Neurol. 2020;10:1-4.

5. Huang YH, Shi QX, Xu MM, Chen CZ, Yang ML, Li JJ, et al. Spinal cord syphilitic gumma presenting with brown-Séquard syndrome: a case report and literature review. Ann Clin Lab Sci. 2019;49:265-70.

6. Shen S, Yang R, Wang L, Tang L, Liu B. Multiple intracranial and spinal cord syphilitic gummas in a human immunodeficiency virus-negative man with untreated syphilis: a case report. Medicine. 2019;98:1-5.

7. Berger JR, Dean D. Neurosyphilis. Handb Clin Neurol. 2014;121: 1461-72.

8. Gonzalez H, Koralnik IJ, Marra CM. Neurosyphilis. Semin Neurol. 2019;39:448-55.

9. Mercurio L, Taylor L, Jarman A. Neurosyphilis: old disease, new implications for emergency physicians. Clin Pract Cases Emerg Med. 2019;4:46-50.

10. Elmouden H, Louhab N, Kissani N. Medullary involvement in neurosyphilis: a report of 12 cases and a review of the literature. Spinal Cord Ser Cases. 2019;5:38.

11. Marra CM. Neurosyphilis. Curr Neurol Neurosci Rep. 2004;4: 435-40.

12. Komamura H, Nakamura T, Kobayashi J, Harada R, Endo K, Ogura $M$, et al. Early neurosyphilis presenting with multiple cranial nerve palsies: a case report of management by combined penicillin-corticosteroid treatment. J Infect Chemother. 2019;25: $362-4$.

13. Ghanem KG. Neurosyphilis: a historical perspective and review. CNS Neurosci Ther. 2010;16:157-68.

14. Xia DY, Zhu MF, Liu CG, Dai Y, Li ZB, Jiang XC, et al. Cerebral syphilitic gumma misdiagnosed as a malignant brain tumor. $\mathrm{J}$ Craniofac Surg. 2017;28:e170-2.

15. Zhou HJ, Zhan RY, Chen MT, Cao F, Zheng XJ. Solitary spinal dural syphilis granuloma mimicking a spinal meningioma. Turk Neurosurg. 2014;24:288-91.

16. Hook EW. Syphilis. Lancet. 2017;389:1550-7.

17. Fadil H, Gonzalez-Toledo E, Kelley BJ, Kelley RE. Neuroimaging findings in neurosyphilis. J Neuroimaging. 2006;16: 286-9.

18. Nagappa M, Sinha S, Taly AB, Rao SL, Nagarathna S, Bindu PS, et al. Neurosyphilis: MRI features and their phenotypic correlation in a cohort of 35 patients from a tertiary care university hospital. Neuroradiology. 2013;55:379-88.

19. Brightbill TC, Ihmeidan IH, Post MJD, Berger JR, Katz DA. Neurosyphilis in HIV-positive and HIV-negative patients: neuroimaging findings. Am J Neuroradiol. 1995;16:703-11.

20. Fargen KM, Alvernia JE, Lin CS, Melgar M. Cerebral syphilitic gummata: a case presentation and analysis of 156 reported cases. Neurosurgery 2009;64:568-75.

21. Shang XJ, He CF, Tang B, Chang XL, Ci C, Sang H. Neuroimaging features, follow-up analyses, and comparisons between asymptomatic and symptomatic neurosyphilis. Dermatol Ther. 2020;10:273-83. 